\title{
Reduced field-of-view diffusion-weighted MRI in patients with cervical cancer
}

\author{
JIYOUNG HWANG, MD, SEONG SOOK HONG, MD, PhD, HYUN-JOO KIM, MD, PhD, YUN-WOO CHANG, MD, PhD, \\ BO DA NAM, MD, EUNSUN OH, MD, EUNJI LEE, MD and HWAJIN CHA, MD
}

Department of Radiology, Soonchunhyang University College of Medicine, Seoul Hospital, Seoul, Republic of Korea

Address correspondence to: Dr Seong Sook Hong

E-mail: hongses@schmc.ac.kr

Objective: Diffusion-weighted imaging (DWI) with reduced field-of-view (FOV) has been shown to provide high spatial resolution with reduced distorsion in the spinal cord, breast, pancreas, and prostate gland. Therefore, we performed this study to evaluate the qualitative image quality and quantitative ADC value of reduced FOV DWI in patients with cervical cancer in comparison with conventional DWI.

Methods: This study retrospectively included 22 patients (mean age, 53.9 years) with biopsy-proven cervical cancer who underwent pelvic MR imaging including conventional DWI and reduced FOV DWI before therapy. Two observers independently rated image quality for reduced FOV DWI and conventional DWI regarding anatomic detail, lesion conspicuity, presence of artifacts, and overall image quality using the following 4-point scale. Quantitative analysis was performed by measuring the ADC value of the tumor. The Wilcoxon signed-rank test was used to compare qualitative scores and mean $A D C$ value between two DWI sequences.

Results: Reduced FOV DWI achieved significantly better anatomic detail, lesion conspicuity, presence of artifacts, and overall image quality compared to conventional DWI $(p<0.05)$. There was no significant difference in mean tumor ADC value between the two DWI sequences $\left(0.990 \times 10^{-3} \mathrm{~mm}^{2} \mathrm{~s}^{-1} \pm 0.364\right.$ at reduced FOV DWI vs $1.253 \times 10^{-3} \mathrm{~mm}^{2} \mathrm{~s}^{-1} \pm 0.387$ at conventional DWI) $(p=0.067)$.

Conclusion: Reduced FOV DWI shows better image quality in terms of anatomic detail and lesion conspicuity with fewer artifacts compared to conventional DWI.

Advance in knowledge: Reduced FOV DWI may enhance diagnostic performance for evaluation of cervical cancer.

\section{INTRODUCTION}

Diffusion-weighted imaging (DWI) shows tissues characteristics based on water diffusion properties, which is related to tissue microenvironment, including tissue cellularity and the integrity of cell membranes. ${ }^{1-3}$ It is well known that tissue apparent diffusion coefficient (ADC) value, which is calculated from DWI, allows quantitative evaluation of tissue diffusivity. ${ }^{4}$ With advances in MR techniques, DWI is widely included as a routine imaging protocol in many organs, including the female pelvis. The clinical application of DWI to cervical cancer has been investigated in many studies. Previous studies demonstrated that ADC value could be useful for differentiating cervical cancer from normal cervix as well as for prediction of the degree and histological type of cervical cancer. ${ }^{5-9}$ Marc et al showed that using DWI resulted in higher reader confidence, sensitivity of tissue infiltration, and tumor-grading for cervical cancer, especially for less experienced reader. ${ }^{10}$ Park et al also reported that tumor $\mathrm{ADC}$ and parametrial invasion on MRI seemed to be independent predictors of pathologic parametrial invasion. Thus, adding DWI to MR imaging would improve accuracy for identifying low-risk patients for parametrial invasion, which is critical for appropriate treatment planning and improvement of patient outcomes. ${ }^{11}$ In addition, DWI might have potential for assessing the therapeutic response to concurrent chemo-radiotherapy (CCRT) in advanced cervical cancer by measuring tumor ADCs or changes in tumor ADCs. ${ }^{12-15}$ These studies were typically performed using single-shot echo-planar imaging (EPI) DWI. Despite these promising results, single-shot EPI has significant limitations; it is prone to susceptibility at air-tissue interfaces and has low spatial resolution. ${ }^{16-19}$ As is well documented in prostate MR imaging in males, susceptibility artifacts at the interface of tissue with air in the rectum is also particularly problematic on DWI of the female uterine cervix. ${ }^{18}$

Recently, DWI sequences with reduced field-of-view (FOV) in the phase-encoding direction have been shown to improve image distortion with high spatial resolution in 
Figure 1. Flow chart of the study population.

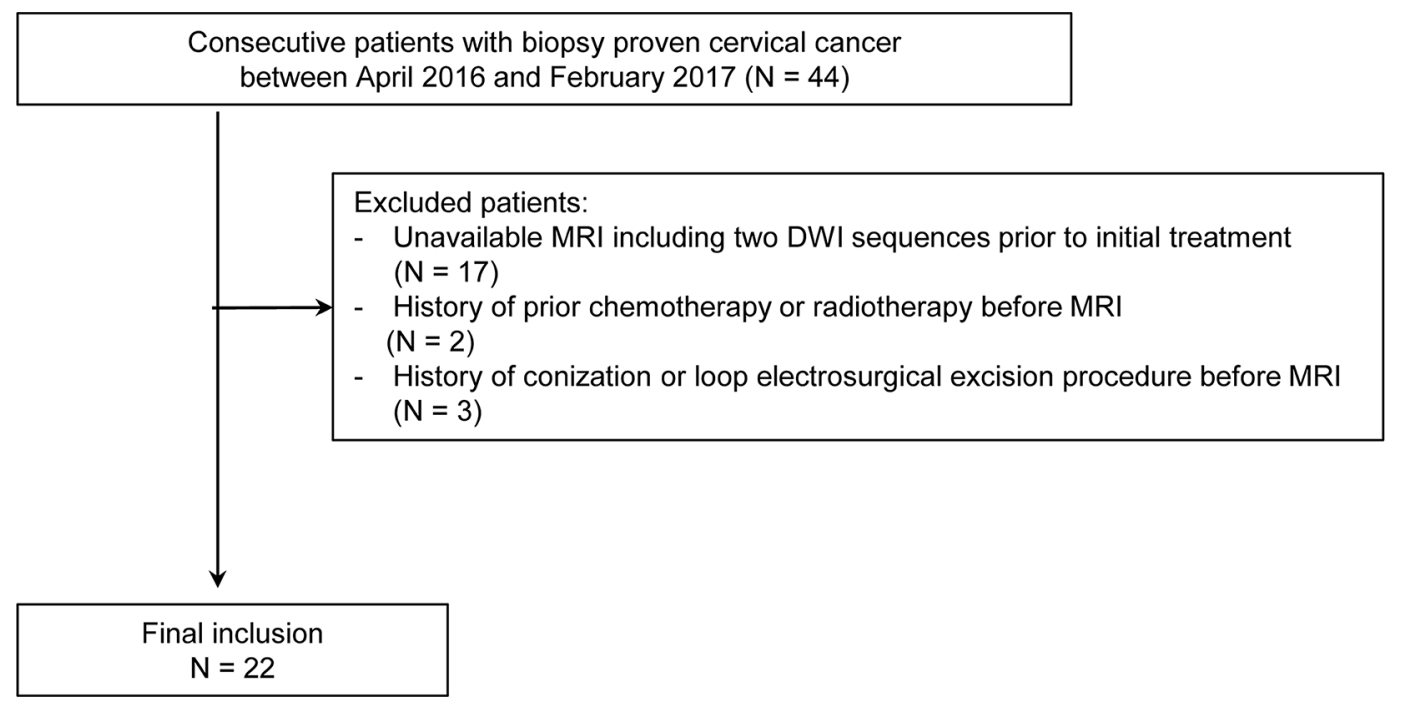

the spinal cord, breast, pancreas, and prostate gland. ${ }^{20-27}$ To the best of our knowledge, application of reduced FOV DWI in the uterine cervix has not been reported. Therefore, we conducted this study to evaluate the qualitative image quality and quantitative ADC values of reduced FOV DWI in patients with cervical cancer in comparison to conventional DWI with $3 \mathrm{~T}$ MRI.

\section{METHODS AND MATERIALS}

\section{Patients}

This retrospective study had institutional review board approval, and informed consent was waived. We retrospectively searched the institutional pathologic database to find patients with biopsy-proven cervical cancer between April 2016 and February 2017. The search identified 44 patients with cervical cancer. The inclusion criteria for the patient group were: (1) biopsy-proven cervical cancer; (2) no history of conization or loop electrosurgical excision procedure before MRI, which could influence MRI interpretation; and (3) pelvic MR imaging including conventional DWI and reduced FOV DWI prior to initial treatment. Thus, a total of 22 patients (mean age, 53.9 years; range, 34-73 years) were included in our study (Figure 1). The clinical stage of cervical cancer was determined by obstetricians who used clinical examination, cystoscopy, and sigmoidoscopy according to the International Federation of Gynecology and Obstetrics classification. ${ }^{28}$ Patients and tumor characteristics are shown in Table 1.

\section{MR examination}

All MR images were acquired using a $3.0 \mathrm{~T}$ MR system (DISCOVERY MR750w; GE Healthcare, Waukesha, WI) with a 32-channel phased-array receiver coil. To reduce bowel peristalsis, $5 \mathrm{mg}$ of cimetropium bromide (Algiron, Boehringer Ingelheim Korea, Cheongju, Korea) was administered intramuscularly before MR examination. There is no consensus in the literature regarding the preparation before MRI including the bowel preparation and use of vaginal/rectal filling with sterile gel, and remains optional. ${ }^{29,30}$ Therefore, bowel preparation or use of vaginal/rectal filling with sterile gel was not performed in our study. Baseline MRI sequences included $T_{1}$ weighted imaging, $T_{2}$ weighted imaging, dynamic contrast-enhanced $T_{1}$ weighted imaging, and DWI. $T_{2}$ weighted fast relaxation fast spin-echo images were obtained in three orthogonal planes (axial, sagittal, and coronal). The imaging parameters of $T_{2} \mathrm{WI}$ were as follows: repetition time (TR) ms/echo time (TE) ms, 3201-5715/80; section thickness, $4 \mathrm{~mm}$; intersection gap, $0.4 \mathrm{~mm}$; matrix, $416 \times 320$; field of view, $28 \mathrm{~cm}$; number of signals acquired, two; reduction factor, two; and acquisition time of each plane, $196 \mathrm{~s}$. Axial $T_{1}$ weighted spin-echo images were obtained to evaluate the lymph nodes and pelvic bone with the following parameters: TR/TE, $643 / 15.0 \mathrm{~ms}$; section thickness, $4 \mathrm{~mm}$; intersection gap, $0.4 \mathrm{~mm}$; field of view, $28 \mathrm{~cm}$; and acquisition time, $188 \mathrm{~s}$. Dynamic contrast-enhanced $T_{1}$ weighted images was obtained using fat-suppressed a three-dimensional (3D) gradient echo sequence in the axial plane (TR/TE, 7.0/3.6 ms; flip angle, 12_; matrix, $256 \times 224$; slice thickness, $4 \mathrm{~mm}$; interslice gap, $2 \mathrm{~mm}$;

Table 1. Patients and tumor characteristics

\begin{tabular}{|c|c|}
\hline No. of patients & 22 \\
\hline $\operatorname{Age}^{a}$ (range) & $53.9 \pm 9.1$ years $(34-73)$ \\
\hline Tumor size $^{a}$ (range) $)^{b}$ & $4.2 \pm 3.2 \mathrm{~cm}(1.5-12.5)$ \\
\hline \multicolumn{2}{|l|}{ FIGO stage (no. of patients) } \\
\hline IA1 & 7 \\
\hline IBI & 9 \\
\hline IB2 & 1 \\
\hline IIB & 4 \\
\hline IVB & 1 \\
\hline \multicolumn{2}{|l|}{ Histologic type } \\
\hline No. of squamous cell carcinoma & 20 \\
\hline No. of adenocarcinoma & 2 \\
\hline
\end{tabular}

FIGO, International Federation of Gynecology and Obstetrics.

a Mean \pm standard deviation.

${ }^{b}$ Tumors that are visible on MRI $(n=15)$. 
Table 2. DWI parameters

\begin{tabular}{|l|c|c|}
\hline Sequence parameter & $\begin{array}{c}\text { Reduced FOV } \\
\text { DWI }\end{array}$ & $\begin{array}{c}\text { Conventional } \\
\text { DWI }\end{array}$ \\
\hline Diffusion directions & $\begin{array}{c}\text { Three-direction } \\
\text { trace }\end{array}$ & $\begin{array}{c}\text { Three-direction } \\
\text { trace }\end{array}$ \\
\hline$b$-value $\left(\mathrm{s} \mathrm{mm}^{-2}\right)$ & 0,800 & 0,1000 \\
\hline Repetition time (ms) & 4000 & 5000 \\
\hline Echo time (ms) & 65 & 69 \\
\hline FOV (cm) & $22 \times 11$ & $30 \times 30$ \\
\hline Matrix & $160 \times 80$ & $140 \times 140$ \\
\hline Section thickness (mm) & 4 & 4 \\
\hline Intersection gap (\%) & 10 & 10 \\
\hline Pixel resolution (mm) & $1.375 \times 1.375$ & $2.143 \times 2.143$ \\
\hline NEX & 16 & 6 \\
\hline Acquisition time (min:s) & $4 \mathrm{~min} 33 \mathrm{sec}$ & $3 \mathrm{~min} 43 \mathrm{sec}$ \\
\hline
\end{tabular}

DWI, diffusion weighted imaging; FOV, field of view; NEX, number of excitations.

FOV, $32 \mathrm{~cm}$; reduction factor, 1.5; NSA, 1; and acquisition time, $5 \mathrm{~min}$ and $2 \mathrm{~s}$ ). A post-contrast series was performed immediately after a bolus injection of Gadoteric acid (Uniray, Dongkook, Korea) at a rate of $1.5 \mathrm{ml} \mathrm{sec}{ }^{-1}$ with a dose of $0.1 \mathrm{mmol}$, followed by a flush of $20 \mathrm{ml}$ of normal saline. DWI was obtained using fat-suppressed, respiratory-triggered echo planar imaging in the axial plane with conventional and reduced FOV. Reduced FOV DWI was performed using a sequence (FOV optimized and constrained undistorted single shot; GE Healthcare, WI) that uses a 2D spatially selective echo-planar radiofrequency excitation pulse and $180^{\circ}$ refocusing pulse to reduce the FOV in the phase-encode direction. The selection of $b$ values ( 0 and 800 or $1000 \mathrm{sec} \mathrm{mm}^{-2}$ ) was made in reference to previous studies. ${ }^{30,31}$ ADC maps were automatically generated with the manufacturer's software. Detailed imaging parameters were listed in Table 2.

\section{Image analysis}

\section{Qualitative analysis}

All images were analyzed independently by two radiologists (with 15 and 7 years of experience in interpreting MR images, respectively) using a picture archiving and communication system. The observers were aware that the patients had biopsy-proven cervical cancer, but were blinded to clinical findings and detailed pathologic results. Two observers subjectively rated image quality for both DWI sequences regarding anatomic detail, lesion conspicuity, artifacts, and overall image quality using the following 4-point scale: (a) anatomic detail, (1) poorly visualized anatomy; (2) fairly delineated anatomic structure with blurred margin; (3) good delineation of anatomic structure with a sharp margin; (4) excellent delineation of anatomic structure; (b) lesion conspicuity, (1) lesion not recognizable; (2) lesion recognizable as slight signal difference; (3) lesion recognizable as distinct signal difference; (4) lesion recognizable as distinct signal difference with a clear lesion margin; (c) presence of artifacts, (1) severe;
(2) moderate; (3) mild; (4) absent; (d) overall image quality, (1) poor image quality, considered non-diagnostic; (2) fair image quality, somewhat impairing diagnostic quality; (3) good image quality, not impairing diagnostic quality; (4) excellent image quality. Cases of invisible tumors on MRI were excluded from assignment of lesion conspicuity. The reviewers analyzed all MR images in an anonymized and randomized manner to minimize bias, in two separate sessions with at least 1-month interval. First, the observers reviewed only conventional DW images. Subsequently, they reviewed reduced FOV DW images using the same criteria. For each DWI sequence, $b=0 \mathrm{~s} \mathrm{~mm}^{-2}$ images were reviewed first, followed by $b=800 \mathrm{~s} \mathrm{~mm}^{-2}$ for reduced FOV and $b=1000 \mathrm{~s} \mathrm{~mm}^{-2}$ for conventional DW images.

\section{Quantitative analysis}

Quantitative analysis was performed by measuring ADC value of the tumor. To obtain the ADC value of the tumor, a circular or elliptical region of interest (ROI) was manually placed on the ADC map to include as much of the tumor as possible in a single image that showed the maximum dimension of visible tumor. $T_{2}$ weighted images were available for recognition of the anatomical details. Care was taken to avoid cystic or necrotic changes within the tumors and to place the ROI in the same position on both sequences. In cases of invisible tumors on MRI, ROIs were placed as large as possible on a central axial plane to include the anterior and posterior epithelial linings of the uterine cervix, according to a previous report. ${ }^{7}$ Tumor ADCs were obtained twice at the same site, and an average was recorded. The mean size of the ROIs was $181 \pm 366 \mathrm{~mm}^{2}$ (range, $80-1846 \mathrm{~mm}^{2}$ ) for conventional images and $178 \pm 429 \mathrm{~mm}^{2}$ (range, $50-2130 \mathrm{~mm}^{2}$ ) for reduced FOV images.

\section{Statistical analysis}

The Wilcoxon signed-rank test was used to compare the qualitative image analysis scores between reduced FOV and conventional DWI sequences. Interobserver agreement for qualitative evaluation was assessed using weighted $\kappa$ statistics. A kappa value less than 0.20 indicates poor agreement; $0.21-0.40$, fair agreement; 0.41-0.60, moderate agreement; 0.61-0.80, good agreement; and greater than 0.81 , excellent agreement. ${ }^{32}$ ADC values of cervical cancer were also compared between the two DWI sequences using the Wilcoxon signed-rank test. Statistical analyses were performed using 2 commercial software programs (MedCalc v. 12.3.0, MedCalc Software, Mariakerke, Belgium; and SPSS 19.0, IBM SPSS Statistics, Armonk, NY). A $p$ value less than .05 was considered significant.

\section{RESULTS}

\section{Qualitative analysis}

Table 3 shows qualitative analysis scores between two DWI. For both observers, reduced FOV DWI achieved significantly better scores in anatomic detail, lesion conspicuity, presence of artifact, and overall image quality at both $b=0 \mathrm{~s} \mathrm{~mm}^{-2}$ and $b=800$ or $1000 \mathrm{~s} \mathrm{~mm}^{-2}$ compared to conventional DWI $(p<0.05)$ (Figures 2 and 3), except for presence of artifact at $b=0 \mathrm{~s} \mathrm{~mm}^{-2}$ with observer $2(p=0.083)$. The mean scores of both observers were also significantly higher on reduced FOV DWI than those on conventional DWI at both $b$ values $(p<0.05)$. Interobserver agreement 
Table 3. Comparison of qualitative analysis scores between reduced FOV DWI and conventional DWI

\begin{tabular}{|c|c|c|c|c|}
\hline & $\begin{array}{c}\text { Anatomic } \\
\text { detail }\end{array}$ & $\begin{array}{c}\text { Lesion } \\
\text { conspicuity }\end{array}$ & $\begin{array}{l}\text { Presence of } \\
\text { artifact }\end{array}$ & $\begin{array}{c}\text { Overall image } \\
\text { quality }\end{array}$ \\
\hline \multicolumn{5}{|l|}{ Observer 1} \\
\hline Reduced FOV DWI $\left(b=0 \mathrm{~s} \mathrm{~mm}^{-2}\right)$ & $3.82 \pm 0.39$ & $3.60 \pm 0.51$ & $3.23 \pm 0.53$ & $3.36 \pm 0.58$ \\
\hline Conventional DWI $\left(b=0 \mathrm{~s} \mathrm{~mm}^{-2}\right)$ & $3.00 \pm 0.31$ & $2.80 \pm 0.41$ & $3.00 \pm 0.62$ & $3.00 \pm 0.44$ \\
\hline$P$ value & $<0.001$ & 0.001 & 0.025 & 0.011 \\
\hline Reduced FOV DWI $\left(b=800 \mathrm{~s} \mathrm{~mm}^{-2}\right)$ & $3.41 \pm 0.50$ & $3.60 \pm 0.51$ & $3.05 \pm 0.58$ & $3.36 \pm 0.49$ \\
\hline Conventional DWI $\left(b=1000 \mathrm{~s} \mathrm{~mm}^{-2}\right)$ & $3.05 \pm 0.38$ & $3.13 \pm 0.52$ & $2.82 \pm 0.59$ & $2.95 \pm 0.38$ \\
\hline$P$ value & 0.005 & 0.008 & 0.025 & 0.003 \\
\hline \multicolumn{5}{|l|}{ Observer 2} \\
\hline Reduced FOV DWI $\left(b=0 \mathrm{~s} \mathrm{~mm}^{-2}\right)$ & $3.68 \pm 0.48$ & $3.47 \pm 0.64$ & $3.00 \pm 0.62$ & $3.32 \pm 0.65$ \\
\hline Conventional DWI $\left(b=0 \mathrm{~s} \mathrm{~mm}^{-2}\right)$ & $3.00 \pm 0.44$ & $2.73 \pm 0.46$ & $2.86 \pm 0.56$ & $2.91 \pm 0.43$ \\
\hline$P \mathrm{p}$ value & $<0.001$ & 0.001 & 0.083 & 0.003 \\
\hline Reduced FOV DWI $\left(b=800 \mathrm{~s} \mathrm{~mm}^{-2}\right)$ & $3.41 \pm 0.59$ & $3.60 \pm 0.51$ & $2.77 \pm 0.69$ & $3.23 \pm 0.61$ \\
\hline Conventional DWI $\left(b=1000 \mathrm{~s} \mathrm{~mm}^{-2}\right)$ & $2.82 \pm 0.59$ & $3.00 \pm 0.53$ & $2.59 \pm 0.59$ & $2.68 \pm 0.57$ \\
\hline$P$ value & 0.001 & 0.007 & 0.046 & 0.001 \\
\hline \multicolumn{5}{|l|}{ Mean } \\
\hline Reduced FOV DWI $\left(b=0 \mathrm{~s} \mathrm{~mm}^{-2}\right)$ & $3.75 \pm 0.44$ & $3.53 \pm 0.57$ & $3.11 \pm 0.58$ & $3.34 \pm 0.61$ \\
\hline Conventional DWI $\left(b=0 \mathrm{~s} \mathrm{~mm}^{-2}\right)$ & $3.0 \pm 0.37$ & $2.77 \pm 0.43$ & $2.93 \pm 0.50$ & $2.95 \pm 0.43$ \\
\hline$P$ value & $<0.001$ & $<0.001$ & 0.005 & $<0.001$ \\
\hline Reduced FOV DWI $\left(b=800 \mathrm{~s} \mathrm{~mm}^{-2}\right)$ & $3.41 \pm 0.54$ & $3.60 \pm 0.50$ & $2.91 \pm 0.64$ & $3.29 \pm 0.55$ \\
\hline Conventional DWI $\left(b=1000 \mathrm{~s} \mathrm{~mm}^{-2}\right)$ & $2.93 \pm 0.50$ & $3.07 \pm 0.52$ & $2.70 \pm 0.59$ & $2.82 \pm 0.49$ \\
\hline$P$ value & $<0.001$ & $<0.001$ & 0.003 & $<0.001$ \\
\hline
\end{tabular}

DWI, diffusion weighted imaging; FOV, field of view.

Data are mean \pm standard deviation.

was fair to excellent on conventional DWI for anatomic detail, lesion conspicuity, presence of artifact, and overall image quality ( $\kappa=0.450-0.815$ at $b=0 \mathrm{~s} \mathrm{~mm} \mathrm{~mm}^{-2}$ and $\kappa=0.388-0.712$ at $b=1000 \mathrm{~s} \mathrm{~mm}^{-2}$ ). There was moderate to excellent agreement between the two observers on reduced FOV DWI for anatomic detail, lesion conspicuity, presence of artifact, and overall image

Figure 2. A 46-year-old female with International Federation of Gynecology and Obstetrics stage IBI adenocarcinoma of the cervix. (a, b) Axial and sagittal $T_{2}$ weighted images show an irregular, intermediate signal intensity cervical mass with intact cervical stromal ring, indicating that the tumor is confined to the cervix. (c-e) Conventional DWI at $b=0 \mathrm{~s} \mathrm{~mm}{ }^{-2}, b=1000 \mathrm{~s} \mathrm{~mm}{ }^{-2}$, and corresponding ADC map with placement of ROI. The ADC value of the lesion is $1.359 \times 10^{-3} \mathrm{~mm}^{2} \mathrm{~s}^{-1}$. (f-h) Reduced FOV DWI at $b=0 \mathrm{~s} \mathrm{~mm} \mathrm{~m}^{-2}, b=800 \mathrm{~s} \mathrm{~mm}^{-2}$, and corresponding ADC map with placement of ROI. The ADC value of the lesion is 1.279 $\times 10^{-3} \mathrm{~mm}^{2} \mathrm{~s}^{-1}$. Compared with conventional DWI, reduced FOV images show the lesion with a clear border and fewer artifacts. ADC, apparent diffusion coefficient; DWI, diffusion-weighted imaging; FOV, field-of-view; ROI, region of interest.
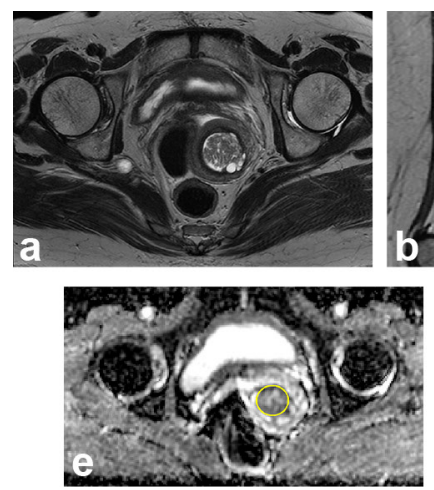
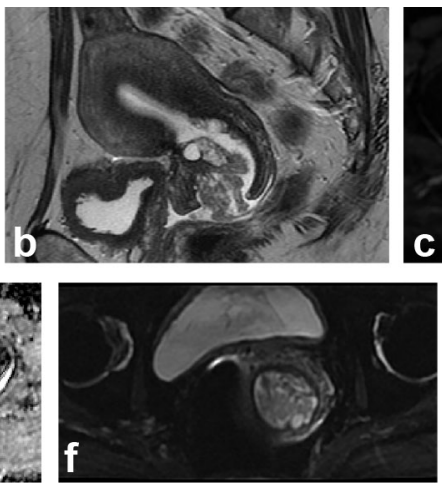
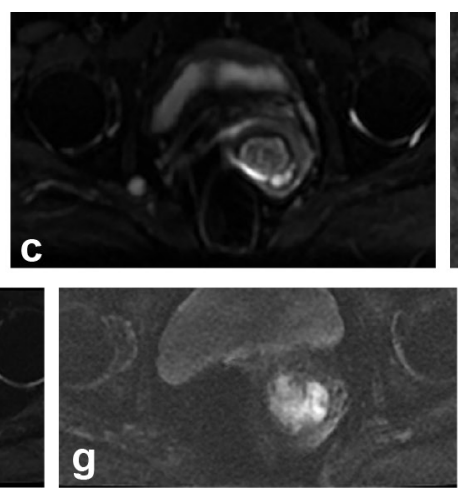
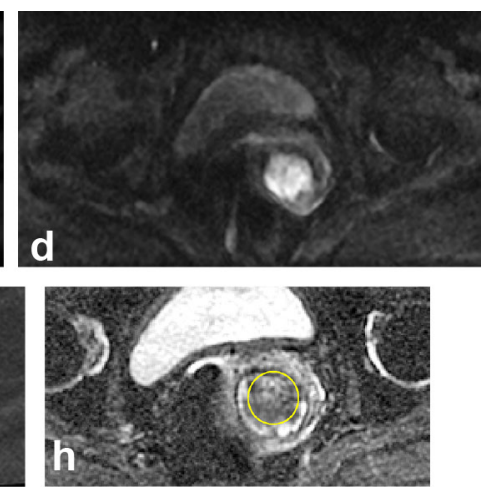
Figure 3. A 58-year-old female with International Federation of Gynecology and Obstetrics stage IBI squamous cell carcinoma of the cervix. (a, b) Axial and sagittal $T_{2}$ weighted images show an intermediate signal intensity cervical mass with interruption of the cervical stromal ring in the right side, without definite extracervical extension (arrow). (c-e) Conventional DWI at $b=0 \mathrm{~s} \mathrm{~mm}^{-2}$, $b=1000 \mathrm{~s} \mathrm{~mm}^{-2}$, and corresponding ADC map with placement of ROI. The ADC value of the lesion is $0.945 \times 10^{-3} \mathrm{~mm}^{2} \mathrm{~s}^{-1}$. ( $\mathrm{f}-\mathrm{h}$ ) Reduced FOV DWI at $b=0 \mathrm{~s} \mathrm{~mm}{ }^{-2}, b=800 \mathrm{~s} \mathrm{~mm}^{-2}$, and corresponding ADC map with placement of ROI. The ADC value of the lesion is $0.915 \times 10^{-3} \mathrm{~mm}^{2} \mathrm{~s}^{-1}$. Compared with conventional DWI, reduced FOV images show the lesion with a clear border and fewer artifacts. ADC, apparent diffusion coefficient; DWI, diffusion-weighted imaging; FOV, field-of-view; ROI, region of interest.
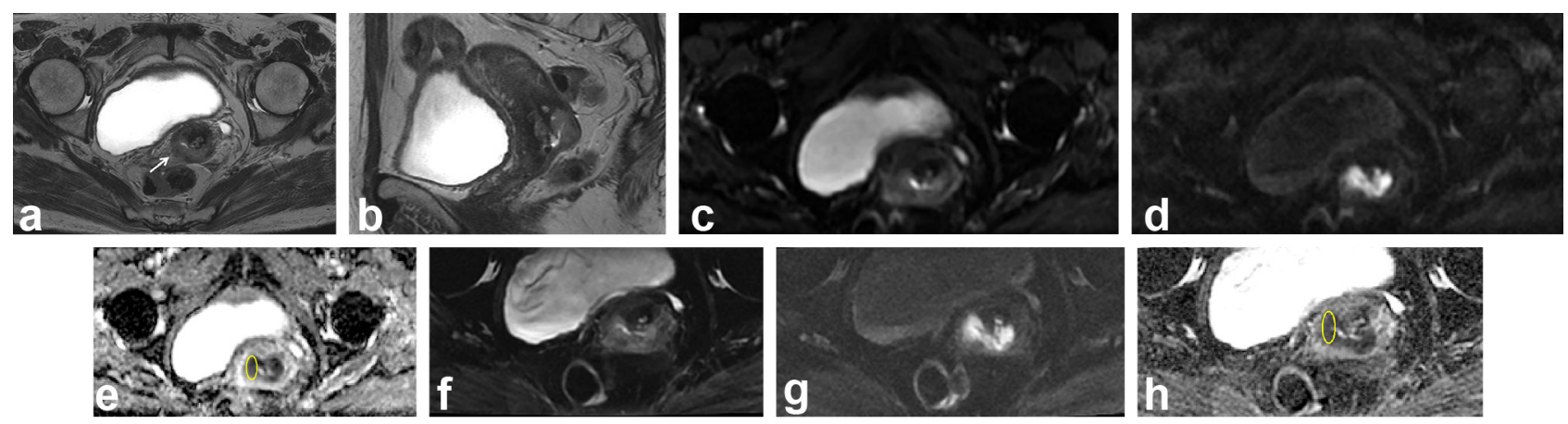

quality $\left(\kappa=0.480-0.762\right.$ at $b=0 \mathrm{~s} \mathrm{~mm}^{-2}$ and $\kappa=0.457-0.831$ at $b=800 \mathrm{~s} \mathrm{~mm}^{-2}$ ) (Table 4$)$.

\section{Quantitative analysis}

There was no significant difference in the mean tumor ADC values between the two DWI sequences $\left(0.990 \times 10^{-3} \mathrm{~mm}^{2} \mathrm{~s}^{-1} \pm 0.364\right.$ on reduced FOV DWI vs $1.253 \times 10^{-3} \mathrm{~mm}^{2} \mathrm{~s}^{-1} \pm 0.387$ on conventional DWI) $(p=0.067)$.

\section{DISCUSSION}

Our results demonstrated that reduced FOV DWI achieved better image quality in terms of anatomic detail and lesion conspicuity compared to conventional DWI. Moreover, image artifacts were decreased on reduced FOV DWI. The reduced FOV DWI has previously been applied to other organs including the pancreas, breast, spinal cord, and prostate gland and has shown improvement of image quality with fewer artifacts. ${ }^{20-27}$ Thus, our study was consistent with the results of previous reports and confirmed that the reduced FOV DWI sequence would have clinical value for cervical cancer. Obtaining DWI of the uterine cervix is particularly challenging due to the anatomical location between the air-containing rectum and urinary bladder, which are prone to susceptibility artifacts and distortion, which can subsequently affect the diagnostic performance, as with DWI of the prostate in males. In this respect, application of reduced FOV DWI on cervical imaging has potential advantages over conventional DWI using single-shot EPI.
Reduced FOV DWI sequence employs a 2-dimensional spatially selective echo-planar radiofrequency pulse and an $180^{\circ}$ refocusing pulse reducing the FOV in the phase-encoding direction instead of conventional excitation in the single-shot EPI sequence. Consequently, this sequence facilitates high spatial resolution imaging with less susceptibility to distortion. ${ }^{24,27}$ Implementation of this sequence also allows decreased partial volume averaging between tumor and normal tissue. ${ }^{22,23}$ The better anatomic detail and lesion conspicuity observed in our study can be explained by high image resolution with decreased partial volume averaging of this technique. We believe this could lead to increased diagnostic performance in identifying small cervical cancer at an early stage, earlier detection of tumor recurrence, more accurate evaluation of tumor extent such as parametrial invasion, and improved assessment of treatment response. Further studies with small cervical cancer at early stage or posttreated tumor are needed in the future.

DWI with large FOV could help to detect pelvic lymph nodes. From this perspective, reduced FOV DWI could be less useful, although not covered in this study. However, according to the European Society of Urogenital Radiology guideline, $T_{1}$ weighted images without fat suppression are useful to evaluate for presence of lymphadenopathy. ${ }^{29}$ Nevertheless, assessment of nodal involvement with cross sectional images that are largely dependent on the size of lymph nodes has significant limitations with low accuracy. Incorporating morphologic features such as

Table 4. Interobserver agreement for qualitative analysis scores

\begin{tabular}{|l|c|c|c|c|}
\hline & Anatomic detail & Lesion conspicuity & Presence of artifact & Overall image quality \\
\hline Reduced FOV $\left(b=0 \mathrm{~s} \mathrm{~mm}^{-2)}\right.$ & $0.645(0.296,0.994)$ & $0.762(0.496,1.000)$ & $0.599(0.307,0.890)$ & $0.480(0.199,0.760)$ \\
\hline Reduced FOV $\left(b=800 \mathrm{~s} \mathrm{~mm}^{-2)}\right.$ & $0.831(0.624,1.000)$ & $0.722(0.364,1.000)$ & $0.457(0.148,0.765)$ & $0.748(0.512,0.984)$ \\
\hline Conventional DWI $\left(b=0 \mathrm{~s} \mathrm{~mm}^{-2)}\right.$ & $0.645(0.196,1.000)$ & $0.815(0.470,1.000)$ & $0.759(0.507,1.000)$ & $0.450(0.020,0.880)$ \\
\hline Conventional DWI $\left(b=1000 \mathrm{~s} \mathrm{~mm}^{-2)}\right.$ & $0.505(0.153,0.856)$ & $0.712(0.344,1.000)$ & $0.626(0.345,0.906)$ & $0.388(0.058,0.700)$ \\
\hline
\end{tabular}

DWI, diffusion weighted imaging; FOV, field of view.

Data are $\kappa$ values.

Data in parentheses are 95\% confidence intervals. 
margin irregularity, heterogeneity of nodal texture, and shape to the size, which are best seen at high-resolution $T_{2}$ weighted MR imaging have shown to be beneficial for differentiating benign and malignant nodes in patients with rectal cancer and may be applicable to those with cervical cancer. ${ }^{30,33}$ Therefore, we think that it is best to use $T_{1}$ weighted images without fat suppression and high-resolution $T_{2}$ weighted imaging for assessing pelvic lymph nodes. Further research on the effect of FOV reduction, particularly regarding assessment of pelvic lymph nodes is needed.

In our study, we found no significant difference in the mean tumor ADC values between two DWI sequences, although reduced FOV sequence had a trend toward lower tumor ADC values. Our results were similar to those of previous reports. ${ }^{20-22,34}$ On the contrary to our results, some studies showed a trend toward lower tumor ADC values for reduced FOV DWI compared to conventional DWI. ${ }^{23,27}$ This difference is believed to be due to reduced partial volume averaging between tumor and normal tissue with the reduced FOV sequence and, consequently, more accurate ADC values. ${ }^{22,23,27}$ In our study, mean tumor ADC values of conventional DWI sequences showed a trend toward higher values than those from the literature, which ranged from 0.757 to $1.11 \times 10^{-3} \mathrm{~mm}^{2} \mathrm{~s}^{-1}$.5,12,35 One possible explanation is that some of our study population had early stage cervical cancer with an invisible tumor compared with those of previous studies. Further, this could be partly explained by the complexity of ADC values that can be affected by multiple factors including $T_{2}, b$ values, signal-to-noise ratio, and partial volume averaging. ${ }^{22,26,36}$
Indeed, mean tumor $\mathrm{ADC}$ values of reduced FOV sequence were within the range of values from other studies. $5,12,35$

This study had several limitations. First, our study included only a small number of patients. Future studies are required with a larger population. Second, the parameters for reduced FOV and conventional DWI sequences were not exactly matched; in particular, the $b$-values of reduced FOV $b=0$ and $800 \mathrm{~s} \mathrm{~mm}^{-2}$ and those of conventional DWI were $b=0$ and $1000 \mathrm{~s} \mathrm{~mm}^{-2}$, which might have affected qualitative and quantitative image analyses. At higher $b$-values, the reduced FOV DWI acquisition would require increased averaging to boost the signal to noise (SNR), subsequently increasing scan time. In fact, it took more time to obtain reduced FOV DWI by increasing averaging to enhance the SNR in our study, which would affect the clinical use in practice. Further effort is needed to reduce scan time at higher $b$-values while maintaining SNR in the future. Third, interobserver variances in qualitative analysis have been reported ${ }^{21,27}$ and also might have affected our results.

In conclusion, reduced FOV DWI showed better image quality in terms of anatomic detail and lesion conspicuity with fewer artifacts compared with conventional DWI in patients with cervical cancer. This could help radiologists to better assess cervical cancer in detail.

\section{FUNDING}

This work was supported by the Soonchunhyang University Research Fund.

\section{REFERENCES}

1. Koh DM, Collins DJ. Diffusion-weighted MRI in the body: applications and challenges in oncology. AJR Am J Roentgenol 2007; 188: 1622-35. doi: https://doi.org/10.2214/AJR. 06.1403

2. Padhani AR, Liu G, Koh DM, Chenevert TL, Thoeny HC, Takahara T, et al. Diffusionweighted magnetic resonance imaging as a cancer biomarker: consensus and recommendations. Neoplasia 2009; 11: 102-25. doi: https://doi.org/10.1593/neo. 81328

3. Hamstra DA, Rehemtulla A, Ross BD. Diffusion magnetic resonance imaging: a biomarker for treatment response in oncology. J Clin Oncol 2007; 25: 4104-9. doi: https://doi.org/10.1200/JCO.2007.11.9610

4. Le Bihan D, Breton E, Lallemand D, Aubin ML, Vignaud J, Laval-Jeantet M. Separation of diffusion and perfusion in intravoxel incoherent motion MR imaging. Radiology 1988; 168: 497-505. doi: https://doi.org/10. 1148/radiology.168.2.3393671

5. Naganawa S, Sato C, Kumada H, Ishigaki T, Miura S, Takizawa O. Apparent diffusion coefficient in cervical cancer of the uterus: comparison with the normal uterine cervix. Eur Radiol 2005; 15: 71-8. doi: https://doi. org/10.1007/s00330-004-2529-4

6. Kaur H, Silverman PM, Iyer RB, Verschraegen CF, Eifel PJ, Charnsangavej C, Diagnosis CC. Diagnosis, staging, and surveillance of cervical carcinoma. AJR Am J Roentgenol 2003; 180: 1621-31. doi: https:// doi.org/10.2214/ajr.180.6.1801621

7. Kuang F, Ren J, Zhong Q, Liyuan F, Huan $\mathrm{Y}$, Chen $\mathrm{Z}$. The value of apparent diffusion coefficient in the assessment of cervical cancer. Eur Radiol 2013; 23: 1050-8. doi: https://doi.org/10.1007/s00330-012-2681-1

8. Hou B, Xiang SF, Yao GD, Yang SJ, Wang YF, Zhang YX, et al. Diagnostic significance of diffusion-weighted MRI in patients with cervical cancer: a meta-analysis. Tumour Biol 2014; 35: 11761-9. doi: https://doi.org/10. 1007/s13277-014-2290-5

9. Park JJ, Kim CK, Park SY, Park BK, Kim B. Value of diffusion-weighted imaging in predicting parametrial invasion in stage IA2-IIA cervical cancer. Eur Radiol 2014; 24:
1081-8. doi: https://doi.org/10.1007/s00330014-3109-x

10. Exner M, Kühn A, Stumpp P, Höckel M, Horn LC, Kahn T, et al. Value of diffusionweighted MRI in diagnosis of uterine cervical cancer: a prospective study evaluating the benefits of DWI compared to conventional MR sequences in a $3 \mathrm{~T}$ environment. Acta Radiol 2016; 57: 869-77. doi: https://doi.org/ 10.1177/0284185115602146

11. Park JJ, Kim CK, Park SY, Park BK. Parametrial invasion in cervical cancer: fused T2-weighted imaging and high-b-value diffusion-weighted imaging with background body signal suppression at 3 T. Radiology 2015; 274: 734-41. doi: https://doi.org/10. 1148/radiol.14140920

12. Chen J, Zhang Y, Liang B, Yang Z. The utility of diffusion-weighted MR imaging in cervical cancer. Eur J Radiol 2010; 74: e101-e106. doi: https://doi.org/10.1016/j. ejrad.2009.04.025

13. Harry VN, Semple SI, Gilbert FJ, Parkin DE. Diffusion-weighted magnetic resonance imaging in the early detection of response to 
chemoradiation in cervical cancer. Gynecol Oncol 2008; 111: 213-20. doi: https://doi.org/ 10.1016/j.ygyno.2008.07.048

14. Park JJ, Kim CK, Park SY, Simonetti AW, Kim E, Park BK, et al. Assessment of early response to concurrent chemoradiotherapy in cervical cancer: value of diffusionweighted and dynamic contrast-enhanced MR imaging. Magn Reson Imaging 2014; 32: 993-1000. doi: https://doi.org/10.1016/j.mri. 2014.05.009

15. Kim HS, Kim CK, Park BK, Huh SJ, Kim B. Evaluation of therapeutic response to concurrent chemoradiotherapy in patients with cervical cancer using diffusion-weighted MR imaging. J Magn Reson Imaging 2013; 37: 187-93. doi: https://doi.org/10.1002/jmri. 23804

16. Mazaheri Y, Vargas HA, Nyman G, Akin $\mathrm{O}$, Hricak H. Image artifacts on prostate diffusion-weighted magnetic resonance imaging: trade-offs at 1.5 Tesla and 3.0 Tesla. Acad Radiol 2013; 20: 1041-7. doi: https:// doi.org/10.1016/j.acra.2013.04.005

17. Tsao J. Ultrafast imaging: principles, pitfalls, solutions, and applications. J Magn Reson Imaging 2010; 32: 252-66. doi: https://doi. org/10.1002/jmri.22239

18. Thian YL, Xie W, Porter DA, Weileng Ang B. Readout-segmented echo-planar imaging for diffusion-weighted imaging in the pelvis at 3T-A feasibility study. Acad Radiol 2014; 21: 531-7. doi: https://doi.org/10.1016/j.acra. 2014.01.005

19. Dietrich O, Biffar A, Baur-Melnyk A, Reiser MF. Technical aspects of MR diffusion imaging of the body. Eur J Radiol 2010; 76: 314-22. doi: https://doi.org/10.1016/j.ejrad. 2010.02.018

20. Ma C, Li YJ, Pan CS, Wang H, Wang J, Chen SY, et al. High resolution diffusion weighted magnetic resonance imaging of the pancreas using reduced field of view single-shot echoplanar imaging at 3 T. Magn Reson Imaging 2014; 32: 125-31. doi: https://doi.org/10. 1016/j.mri.2013.10.005

21. Kim H, Lee JM, Yoon JH, Jang JY, Kim SW, Ryu JK, et al. Reduced field-of-view diffusion-weighted magnetic resonance imaging of the pancreas: comparison with conventional single-shot echo-planar imaging. Korean J Radiol 2015; 16: 1216-25. doi: https://doi.org/10.3348/kjr.2015.16.6. 1216

22. Singer L, Wilmes LJ, Saritas EU, Shankaranarayanan A, Proctor E, Wisner DJ, et al. High-resolution diffusion-weighted magnetic resonance imaging in patients with locally advanced breast cancer. Acad Radiol 2012; 19: 526-34. doi: https://doi.org/10. 1016/j.acra.2011.11.003

23. Dong H, Li Y, Li H, Wang B, Hu B. Study of the reduced field-of-view diffusion-weighted imaging of the breast. Clin Breast Cancer 2014; 14: 265-71. doi: https://doi.org/10. 1016/j.clbc.2013.12.001

24. Saritas EU, Cunningham CH, Lee JH, Han ET, Nishimura DG. DWI of the spinal cord with reduced FOV single-shot EPI. Magn Reson Med 2008; 60: 468-73. doi: https://doi. org/10.1002/mrm.21640

25. Andre JB, Zaharchuk G, Saritas E, Komakula S, Shankaranarayan A, Banerjee S, et al. Clinical evaluation of reduced field-of-view diffusion-weighted imaging of the cervical and thoracic spine and spinal cord. AJNR Am J Neuroradiol 2012; 33: 1860-6. doi: https:// doi.org/10.3174/ajnr.A3134

26. Feng Z, Min X, Sah VK, Li L, Cai J, Deng M, et al. Comparison of field-of-view (FOV) optimized and constrained undistorted single shot (FOCUS) with conventional DWI for the evaluation of prostate cancer. Clin Imaging 2015; 39: 851-5. doi: https://doi.org/ 10.1016/j.clinimag.2015.03.004

27. Korn N, Kurhanewicz J, Banerjee S, Starobinets O, Saritas E, Noworolski S. Reduced-FOV excitation decreases susceptibility artifact in diffusion-weighted MRI with endorectal coil for prostate cancer detection. Magn Reson Imaging 2015; 33: 56-62. doi: https://doi.org/10.1016/j.mri. 2014.08.040

28. Pecorelli S, Zigliani L, Odicino F. Revised FIGO staging for carcinoma of the cervix. Int J Gynaecol Obstet 2009; 105: 107-8. doi: https://doi.org/10.1016/j.ijgo.2009.02.009

29. Balleyguier C, Sala E, Da Cunha T, Bergman A, Brkljacic B, Danza F, et al. Staging of uterine cervical cancer with MRI: guidelines of the European Society of Urogenital Radiology. Eur Radiol 2011; 21: 1102-10. doi: https://doi.org/10.1007/s00330-010-1998-x

30. Rauch GM, Kaur H, Choi H, Ernst RD, Klopp AH, Boonsirikamchai P, et al. Optimization of MR imaging for pretreatment evaluation of patients with endometrial and cervical cancer. Radiographics 2014; 34: 1082-98. doi: https:// doi.org/10.1148/rg.344140001

31. Noël P, Dubé M, Plante M, St-Laurent G. Early cervical carcinoma and fertility-sparing treatment options: MR imaging as a tool in patient selection and a follow-up modality. Radiographics 2014; 34: 1099-119. doi: https://doi.org/10.1148/rg.344130009

32. Landis JR, Koch GG. An application of hierarchical kappa-type statistics in the assessment of majority agreement among multiple observers. Biometrics 1977; 33: 363-74. doi: https://doi.org/10.2307/2529786

33. Brown G, Richards CJ, Bourne MW, Newcombe RG, Radcliffe AG, Dallimore NS, et al. Morphologic predictors of lymph node status in rectal cancer with use of high-spatial-resolution MR imaging with histopathologic comparison. Radiology 2003; 227: 371-7. doi: https://doi.org/10.1148/ radiol.2272011747

34. Zaharchuk G, Saritas EU, Andre JB, Chin CT, Rosenberg J, Brosnan TJ, et al. Reduced field-of-view diffusion imaging of the human spinal cord: comparison with conventional single-shot echo-planar imaging. AJNR Am J Neuroradiol 2011; 32: 813-20. doi: https:// doi.org/10.3174/ajnr.A2418

35. Levy A, Medjhoul A, Caramella C, Zareski E, Berges O, Chargari C, et al. Interest of diffusion-weighted echo-planar MR imaging and apparent diffusion coefficient mapping in gynecological malignancies: a review. J Magn Reson Imaging 2011; 33: 1020-7. doi: https://doi.org/10.1002/jmri. 22546

36. Saritas EU, Lee JH, Nishimura DG. SNR dependence of optimal parameters for apparent diffusion coefficient measurements. IEEE Trans Med Imaging 2011; 30: 424-37. doi: https://doi.org/10.1109/TMI.2010. 2084583 